\title{
Ferulic acid and its derivatives modulate nitric oxide balance in HUVEC cells
}

\author{
M. Le Sayec ${ }^{1}$, G. Serreli ${ }^{1}$, E. Thou ${ }^{1}$, C. Lacour ${ }^{1}$, J.P.E. Spencer ${ }^{2}$ and G. Corona ${ }^{1}$ \\ ${ }^{1}$ Health Sciences Research Centre, Life Sciences Department, Whitelands College, \\ University of Roehampton, London, UK and \\ ${ }^{2}$ Molecular Nutrition Group, School of Chemistry, Food and Pharmacy, University of Reading, \\ Reading RG6 6AP, UK
}

Nitric oxide (NO) is an important vasodilator involved in the regulation of vascular homeostasis, and plays a crucial role in maintaining a normal endothelial function ${ }^{(1)}$. Dietary phenolic acids such as ferulic acid can improve vascular function ${ }^{(2)}$, through maintenance of local levels of NO. Thus the molecular events involved are not fully understood ${ }^{(3)}$ and further mechanistic investigations are required. The aim of this study is to understand the precise cellular mechanisms by which ferulic acid and its metabolites maintain healthy vascular function through modulation of the NO pathway.

Primary Human Umbilical Vein Endothelial Cells were exposed to ferulic acid, isoferulic acid, hydroferulic acid, ferulic acid 4-O-glucuronide, isoferulic Acid 3-O-Sulfate and Dihydroferulic acid 4-O-glucuronide $(1 \mu \mathrm{M})$ for $24 \mathrm{~h}$ or $2 \mathrm{~h}$. Apocynin and $\mathrm{N} \omega$-Nitro-L-arginine (L-NNA) were used as additional controls. Superoxide production, cyclic GMP cGMP) levels, and Akt1 activation were determined. The statistical analysis of the data (at least 4 biological replicates) was conducted by one-way ANOVA followed by a Tukey post-hoc t-test using the Graphpad 7 software.

\begin{tabular}{|c|c|c|c|}
\hline Cell treatments & $\begin{array}{c}\text { cyclic GMP } \\
\text { cGMP (pmol/mg prot) }\end{array}$ & $\begin{array}{l}\text { Superoxide release } \\
(\% \text { of control })\end{array}$ & $\begin{array}{c}\text { Aktl } \\
\text { ph/tot ratio }\end{array}$ \\
\hline Control & $5 \cdot 12 \pm 0 \cdot 49$ & $100 \pm 1.90$ & $1 \cdot 00 \pm 0 \cdot 15$ \\
\hline Apocynin $(100 \mu \mathrm{M})$ & $15.71 \pm 1.17 * *$ & $66.95 \pm 2.07 * * *$ & $1.52 \pm 0.17 * *$ \\
\hline $\mathrm{N} \omega$-Nitro-L-arginine L-NNA $(100 \mu \mathrm{M})$ & $3 \cdot 58 \pm 0 \cdot 32$ & $166 \cdot 2 \pm 4.66 * * *$ & $0 \cdot 81 \pm 0 \cdot 09$ \\
\hline Ferulic acid $(1 \mu \mathrm{M})$ & $21.94 \pm 2.04 * * *$ & $77.69 \pm 1.47 *$ & $1.53 \pm 0.13 * * *$ \\
\hline Isoferulic acid $(1 \mu \mathrm{M})$ & $20 \cdot 89 \pm 1.93 * * *$ & $69.06 \pm 2.46 * * *$ & $1.72 \pm 0.09 * * *$ \\
\hline Hydroferulic Acid $(1 \mu \mathrm{M})$ & $29 \cdot 55 \pm 1.19 * * *$ & $61 \cdot 29 \pm 2 \cdot 37 * * *$ & $1.43 \pm 0.13 * *$ \\
\hline Ferulic acid 4-O-glucuronide $(1 \mu \mathrm{M})$ & $16 \cdot 5 \pm 2 \cdot 60 *$ & $73 \cdot 35 \pm 3 \cdot 84 * *$ & $1.26 \pm 0.06$ \\
\hline Isoferulic Acid 3-O-Sulfate $(1 \mu \mathrm{M})$ & $17 \cdot 66 \pm 3.24 * *$ & $74 \cdot 44 \pm 3 \cdot 31 * *$ & $1 \cdot 52 \pm 0.22 * *$ \\
\hline Hydroferulic acid 4-O-glucuronide $(1 \mu \mathrm{M})$ & $17.85 \pm 2.67 * *$ & $66.08 \pm 2.75 * * *$ & $1 \cdot 24 \pm 0 \cdot 12$ \\
\hline
\end{tabular}

$*=\mathrm{p}<0 \cdot 05, * *=\mathrm{p}<0 \cdot 01, * * *=\mathrm{p}<0.001$ vs. control

Similarly to apocynin, all compounds tested showed the ability to enhance NO levels, measured as production of cGMP, and significantly decrease superoxide production, Protein expression results obtained through western blotting showed that the treatment with ferulic acid, isoferulic acid, hydroferulic acid and isoferulic Acid 3-O-Sulfate was also able to significantly increase Akt1 activation, measured as the ratio of the phosphorylated (Ser 473) / total protein. Our results indicated that all the tested ferulic acid metabolites can modulated NO balance, measured as cGMP production, by decreasing its degradation (via reduced superoxide formation), however the glucuronide-conjugated metabolites are not able to significantly enhance NO production through the Akt1/eNOS pathway.

This work was supported by the BBSRC (BB/M002802/1)

1. Leong XF et al. (2015) Biomed Res Int May (3). 528757.

2. Vauzour D et al. (2010) Nutrients (2): 1106-1131.

3. Corona G, et al. (2014) Nutrition and Aging 2(2-3): 125-132. 